SLAC-PUB-14839

December 2011

\title{
TERAHERTZ RADIATION FROM A PIPE WITH SMALL CORRUGATIONS ${ }^{\mathrm{a}}$
}

\author{
K.L.F. Bane and G. Stupakov \\ SLAC National Accelerator Laboratory, \\ Stanford University, Stanford, CA 94309
}

\begin{abstract}
We have studied through analytical and numerical methods the use of a relativistic electron bunch to drive a metallic beam pipe with small corrugations for the purpose of generating terahertz radiation. For the case of a pipe with dimensions that do not change along its length, we have shown that - with reasonable parameters - one can generate a narrow-band radiation pulse with frequency $\sim 1 \mathrm{THz}$, and total energy of a few milli-Joules. The pulse length tends to be on the order of tens of picoseconds. We have also shown that, if the pipe radius is tapered along its length, the generated pulse will end up with a frequency chirp; if the pulse is then made to pass through a compressor, its final length can be reduced to a few picoseconds and its peak power increased to $1 \mathrm{GW}$. We have also shown that wall losses tend to be significant and need to be included in the structure design.
\end{abstract}

${ }^{a}$ Work supported by Department of Energy contract DE-AC02-76SF00515. 


\section{INTRODUCTION}

For applications in fields as diverse as chemical and biological imaging, material science, telecommunication, semiconductor and superconductor research, there is great interest in having a source of short, intense pulses of terahertz radiation. There are laser-based sources of such radiation [1, 2], capable of generating e.g. (several-cycle) pulses with frequency over the range 10-70 THz and energy of $20 \mu \mathrm{J}[3]$. And there are beam-based sources, utilizing short, relativistic electron bunches $[4,5]$. One beam-based method impinges an electron bunch on a thin metallic foil and generates coherent transition radiation (CTR). Recent tests of this method at the Linac Coherent Light Source (LCLS) have obtained single-cycle pulses of radiation that is broadband, centered on $10 \mathrm{THz}$, and contains $>0.1 \mathrm{~mJ}$ of energy [6]. Another beam-based method generates $\mathrm{THz}$ radiation by passing a bunch through a metallic pipe with a dielectric layer [7]. At UCLA this method was used to generate narrow-band pulses with frequency $0.4 \mathrm{THz}$ and energy $10 \mu \mathrm{J}$. In this report we investigate a similar idea, that of using a short, relativistic beam to generate $\mathrm{THz}$ radiation in a metallic pipe with small corrugations, to explore what the possibilites of this approach might be.

It has been noted in the past, in the study of wall-roughness impedance, that a beam passing through a metallic pipe with small-scale corrugations excites a high-frequency mode that propagates with the beam. We propose introducing a short driving bunch into such structure, with aperture on the order of a millimeter and length on the order of a meter, in order to generate a pulse of radiation with frequency on the order of a terahertz and energy on the order of milli-Joules. With a pipe where the corrugations do not vary along the structure, we shall see that we obtain a narrow-band pulse that is relatively long, on the order of tens of picoseconds. To obtain a shorter pulse, 
one can imagine using a corrugated pipe that varies along its length, in order to introduce a frequency chirp in the pulse. If this is followed by a properly designed dispersive device - analogous to what is done in chirped pulse amplification (CPA) in high power lasers - the pulse can then be compressed, at the expense of being more broad-band than in the previous, unchirped case.

In this report we will study both ideas, $\mathrm{THz}$ pulse generation in a corrugated pipe that does not vary along its length and in one that does. In the second case, however, we will not attempt to solve the entire problem. We will focus on the first part of the process - generating the chirped pulse. Pulse generation in a corrugated pipe is studied using analytical formulas and numerical simulations. For the simulations we employ I. Zagorodnov's program ECHO, which computes the fields generated by an ultra-relativistic bunch in a structure in the time domain [8]. We begin with lossless beam pipe walls, and then include wall losses in the calculations. The pulse compression that follows is only treated conceptually, by simulating the effect of an ideal dispersive compressor. It is understood however, that working out an actual design for such a compressor will be a crucial component in getting a short, high peak power $\mathrm{THz}$ pulse from a pipe with small corrugations.

\section{THEORY}

\section{A. Analytical estimates}

Consider a short, ultra-relativistic bunch of electrons passing on-axis through a periodic (cylindrically-symmetric) metallic structure with small corrugations. Let the pipe radius be $a$, and the corrugations have depth $\delta$, period $p$, and gap $g$, with $\delta, p \ll a$ (see Fig. 1). When $\delta \gtrsim p$ (a case that we call "steeply corrugated") the beam excites one dominant mode at a fre- 
quency that is far above cut-off, in a pulse that follows the beam near the speed of light, $c$ (many higher frequency weak modes are also excited) ${ }^{1}$. For the dominant, fundamental mode the wave number, $k$, and group velocity, $v_{g}$, are well approximated by $[9,10]$

$$
\begin{gathered}
k=\frac{2}{\sqrt{a \delta}}, \\
\left(1-\frac{v_{g}}{c}\right)=\frac{2 \delta}{a} \ll 1 .
\end{gathered}
$$

Here, and in the rest of the report, for simplicity we have let the gap $g=p / 2$ (for the general case, when this relation does not hold, the analytical formulas can be found in Refs. $[9,10])$.

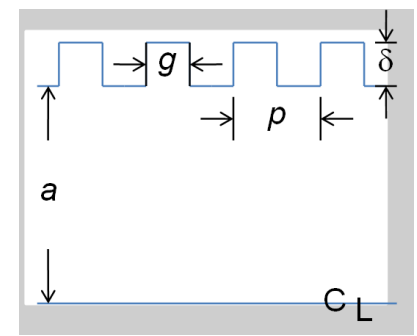

FIG. 1. A sketch of a part of the corrugated structure.

A schematic of how the pulse is generated, and how it would arrive at a monitor located at the structure end is shown in Fig. 2. As the beam (the blue elliptical symbol in frames a-c) traverses the structure, parts of a radiation pulse are continually being generated by it and then fall behind (the blue, green, and red stripes represent parts of the radiation pulse created, respectively, at the beginning, middle, and end of the structure). Finally, at the field monitor the parts of the pulse arrive in the reverse order in which

\footnotetext{
${ }^{1}$ Note that if the beam moves through the structure slightly off axis, a dominant dipole mode of the same frequency will also be excited.
} 
they were created. If the pipe length is $L$, then Eq. 2 implies that the length of the radiation pulse at the downstream end is

$$
\ell=\frac{2 \delta L}{a} .
$$
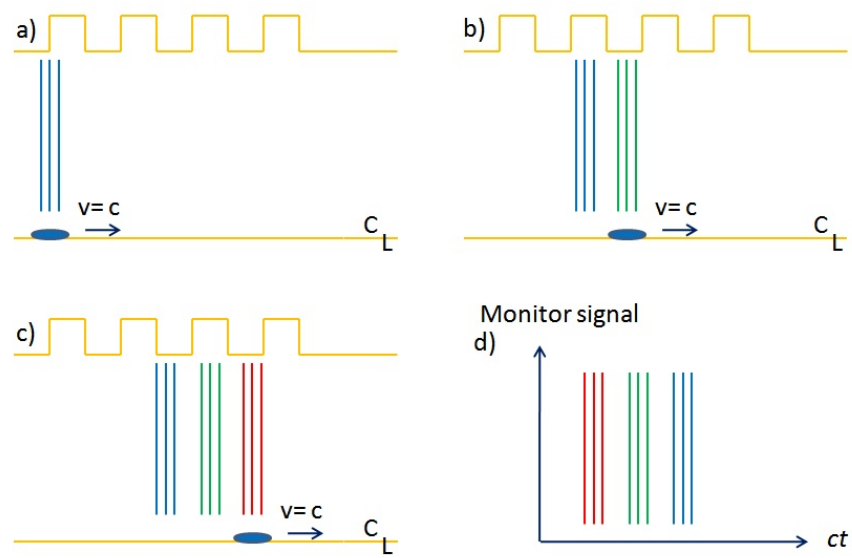

FIG. 2. Schematic of: pulse generation by bunch in corrugated beam pipe (a-c), and signal measured at a monitor at the end of the structure $(d)$.

Let us assume, for the moment, that the walls are perfectly conducting. Then the energy in the pulse at the end of the corrugated pipe is approximately equal to the energy lost by the exciting bunch during its passage through the pipe. And this loss, in turn, is approximately given by the loss to the fundamental mode alone (assuming a steeply corrugated structure, i.e. $\delta \gtrsim p$ ). (In the simulations, to be presented below, we will confirm these statements.) The energy loss (or "wake loss") is given by $U_{w}=Q^{2} \kappa L$, with $Q$ the charge in the bunch and $\kappa$ the loss factor [9-11]:

$$
\kappa=\frac{Z_{0} c}{2 \pi a^{2}} e^{-k^{2} \sigma_{z}^{2}}
$$

where $Z_{0}=377 \Omega$ and $\sigma_{z}$ is the rms bunch length. Note that with a very short bunch $\left(k \sigma_{z} \ll 1\right), \kappa=Z_{0} c / 2 \pi a^{2}$, and the wake loss is the largest (steadystate) loss in any structure with minimum aperture $a$ and length $L$ (see e.g. 
discussion in Ref. [12]). The peak power in the pulse is then given by $P=$ $2 c U_{w} / \ell$.

As a practical example, consider a beam pipe with $a=2 \mathrm{~mm}, \delta=50 \mu \mathrm{m}$, $p=40 \mu \mathrm{m}, L=50 \mathrm{~cm}$, and an exciting bunch with $\sigma_{z}=100 \mu \mathrm{m}, Q=$ $1 \mathrm{nC}$. For this example, the analytical formulas give: frequency $f=k c / 2 \pi$ $=0.3 \mathrm{THz} ; k \sigma_{z}=0.63$, bunch energy loss $U_{w}=1.5 \mathrm{~mJ}$ (for a point charge the loss would be $U_{w}=2.25 \mathrm{~mJ}$ ); peak power $P=30 \mathrm{MW}$; and final pulse length $\ell=2.5 \mathrm{~cm}$. We see that the frequency is on the order of $1 \mathrm{THz}$, the energy in the pulse is significant, and the pulse is quite long.

Some notes to consider concerning the results of this section:

1. All of our analytical results are valid for the case of a steeply corrugated structure $(\delta \gtrsim p)$. If this condition is slightly violated, the mode frequency will be higher and the excitation (loss factor) lower than the analytical values. In the regime of a "shallowly corrugated" structure $(\delta \ll p)$ the dominant mode is gone, and the structure has a completely different behavior.

2. The results given here are steady-state results. When a beam first enters a corrugated pipe there is a different, transient response that we have ignored. After a distance on the order of the catch-up distance, $z_{c u}=$ $a^{2} / 2 \sigma_{z}$, the analytical formulas become valid. We will see that for the size of parameters discussed in this report, the transient distance is relatively small and transient effects can indeed be ignored.

3. The radiation pulse generated by the corrugated structure will be cylindrically symmetric of radius $a$. The fields vary linearly with radius: $E_{r}=H_{\phi}=H_{0} r / a$, with $E_{r}$ the radial electric field, $H_{\phi}$ the azimuthal magnetic field, and $H_{0}$ a constant [13]. 
4. Resistive wall losses, which are ignored here, will significantly affect the pulse energy and peak power that can be achieved. This important issue will be addressed in a later section of this report.

\section{B. Tapered Structure}

The pulse power can be enhanced if it is compressed to a fraction of a centimeter. This can be achieved by generating a terahertz pulse whose frequency varies from head to tail, and sending it through a dispersive system in which the head travels a shorter path than the tail. We will call such a system a compressor. Mathematical representation of the compressor action of the pulse is discussed in the next subsection.

To introduce a frequency chirp into the pulse, we have considered adiabatically varying the corrugation parameters along the pipe. One of the simplest methods is to keep the actual corrugations unchanged, and to just vary the beam pipe radius gradually. Fig. 3 gives a sketch of the idea: the beam passes through a pipe that becomes gradually smaller. At the end there is a monitor to measure the pulse. This is followed by, at the moment, an ideal pulse compressor. In the configuration shown the front of the pulse will have a higher frequency than the back. However, the inverse configuration-small-to-large beampipe - should, in principle work as well.

The parameters for the tapered, corrugated structure and for the exciting bunch to be used in simulations presented below are given in Table I. In particular, the pipe radius varies linearly from $a=2 \mathrm{~mm}$ in the beginning to $1 \mathrm{~mm}$ at the end. The taper is gradual, so we assume the equation for $k$ (Eq. 1) is valid locally; averaging along the structure, we estimate the central frequency to be $0.35 \mathrm{THz}$, and the bandwidth $0.125 \mathrm{THz}$. Note that estimating the final radiation pulse length is not so easy, and requires a detailed knowledge of the 


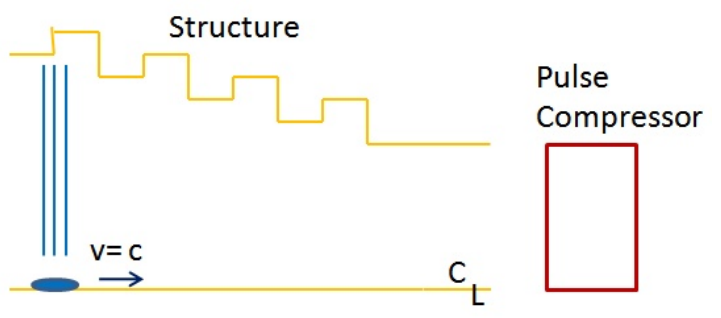

FIG. 3. Schematic of pulse generation by bunch in tapered, corrugated beam pipe, followed by pulse compression. The front of the pulse measured at the monitor will have higher frequency than the back.

dispersion curve of a corrugated pipe - something that is beyond the scope of this report.

TABLE I. Parameters for the standard tapered, corrugated structure and for the exciting bunch, to be used in simulations given in the next section.

\begin{tabular}{||l|c||}
\hline \hline Pipe radius $a, \mathrm{~mm}$ & $2-1$ \\
Pipe length $L, \mathrm{~cm}$ & 50 \\
Depth $\delta, \mu \mathrm{m}$ & 50 \\
Period $p, \mu \mathrm{m}$ & 40 \\
Gap $g, \mu \mathrm{m}$ & 20 \\
Bunch charge $Q, \mathrm{nC}$ & 1 \\
Bunch length $\sigma_{z}, \mu \mathrm{m}$ & 100 \\
\hline \hline
\end{tabular}

The analytical approximation of the energy in the radiation pulse, given by the wake energy, is $U_{w}=Q^{2} L\langle\kappa\rangle$, with $\kappa$ given in Eq. 4 and the brackets means to average along the length of the pipe. For our linear taper the average can be performed explicitly, giving

$$
U_{w}=\frac{Z_{0} c}{8 \pi} \frac{Q^{2} L \delta}{\sigma_{z}^{2}\left(a_{f}-a_{0}\right)}\left[\exp \left(\frac{-4 \sigma_{z}^{2}}{a_{f} \delta}\right)-\exp \left(\frac{-4 \sigma_{z}^{2}}{a_{0} \delta}\right)\right],
$$


with $a_{0}\left(a_{f}\right)$ the initial (final) pipe radius.

\section{Pulse Compression}

The pulse generated in the tapered structure will have a frequency chirp. For compression the pulse then needs to traverse a properly designed dispersive device or region, a compressor.

Without actually designing the compressor we can formulate the action of the compressor on the pulse. The transverse electric field of a pulse with a linear chirp and a Gaussian envelope can be written as (see e.g. [14])

$$
E(t)=E_{0} \exp \left[-\alpha t^{2}\right] \exp \left[i\left(\omega_{0} t-\beta t^{2}\right)\right]+\text { c.c. },
$$

with $\omega_{0}$ the central frequency. The rms pulse length $\sigma_{z p}=c / \sqrt{2 \alpha}$ and the frequency chirp $d \omega / d t=-2 \beta$. The Fourier transform of the field is given by

$$
\tilde{E}(\omega)=E_{0} \exp \left[-\frac{\alpha / 4}{\alpha^{2}+\beta^{2}}\left(\omega-\omega_{0}\right)^{2}\right] \exp \left[-i \frac{\beta / 4}{\alpha^{2}+\beta^{2}}\left(\omega-\omega_{0}\right)^{2}\right],
$$

where the result has been factored into amplitude and phase parts. Note that the phase $\arg (\tilde{E})$ is proportional to $\left(\omega-\omega_{0}\right)^{2}$.

Passage of the pulse through an ideal dispersive medium adds an additional phase $\phi(\omega)$ to the electric field (7) without changing its amplitude. If this phase is chosen in such a way that it cancels the phase in Eq. 7, the resulting pulse length becomes $\sigma_{z \text {,comp }}=c \sqrt{\alpha / 2\left(\alpha^{2}+\beta^{2}\right)}$. We find that the ratio of pulse length before to after compression - the compression factor - is given by

$$
f_{c}=\frac{\sigma_{z p}}{\sigma_{z, \mathrm{comp}}}=\frac{\sqrt{\alpha^{2}+\beta^{2}}}{\alpha},
$$

which in the limit of large enough chirp, $\beta \gg \alpha$, is approximately equal to $\beta / \alpha$ and is much larger than one. 
Let us make a rough estimate of the relative sizes of $\alpha, \beta$, and the compression one can expect for the nominal tapered structure (a more detailed analysis based on our simulations is presented in Section III). Suppose the initial pulse envelope is relatively uniform with length $\ell=4 \mathrm{~cm}$ (which we will see in simulations below), meaning that $\sigma_{z p}=1.2 \mathrm{~cm}$. The chirp parameter can be approximated $\beta \sim c^{2}\left[k\left(a_{0}\right)-k\left(a_{f}\right)\right] / 2 \ell$, with $a_{0}\left(a_{f}\right)$ the initial (final) beam pipe radius. Then $\alpha / c^{2}=0.004 \mathrm{~mm}^{-2}$ and $\beta / c^{2}=0.033 \mathrm{~mm}^{-2}$, much larger. This gives for the compression factor $f_{c} \sim 8$.

Note that a simple dispersive medium for an electromagnetic pulse is represented by a straight round pipe with smooth conducting walls. The lowest $\mathrm{TM}_{01}$ mode in such a pipe with frequency $\omega$ has a longitudinal wavenumber

$$
k_{z}=\frac{\omega}{c}-\frac{c \mu_{01}^{2}}{2 a^{2} \omega},
$$

where $a$ is the radius of the pipe, $\mu_{01}$ is the first root of the zero order Bessel function, and we assumed that the frequency $\omega$ is well above of the cutoff frequency $c \mu_{01} / a$. It is easy to see that for a wave packet with a small frequency spread around $\omega_{0}$, a passage of the packet through a pipe of length $L$ introduces a phase difference whose quadratic (in $\omega-\omega_{0}$ ) part is

$$
\left.\phi \approx \frac{1}{2} L\left(\omega-\omega_{0}\right)^{2} \frac{d^{2} k_{z}}{d \omega^{2}}\right|_{\omega=\omega_{0}}=-L\left(\omega-\omega_{0}\right)^{2} \frac{c \mu_{01}^{2}}{a^{2} \omega_{0}^{3}},
$$

which in principle can be used to compensate for the frequency chirp in (7). Unfortunately, numerical estimates show that, due to the high frequency of terahertz radiation and for reasonable values of radius $a$, the dispersive effect of a smooth pipe is tiny. To use such a pipe for compression would require it to be extremely long (in which case the wall losses would no longer be negligible). Even if one uses a low-loss pipe with periodic irises [15] (which, in the lowest order has the same dispersive properties as a smooth pipe with radius equal to the iris radius) the required length does not seem practical. 
We believe that a practical compressor should be based on diffraction gratings and mirrors, similar to ones used in optics for CPA. The detailed design of such a compressor is, however, beyond the scope of this paper.

\section{SIMULATIONS}

We perform numerical simulations using I. Zagorodnov's 2D version of the time-domain, finite difference Maxwell equation solver ECHO [8]. The program finds wakefields excited by a speed-of-light Gaussian bunch that passes through a metallic structure, such as a corrugated beam pipe. The program was recently modified to allow for "monitors", chosen locations within the structure where one can record the electric and magnetic fields as functions of time.

Our first simulation is for the $50 \mathrm{~cm}$ long corrugated pipe of Table I, but without the taper, keeping the pipe radius at $a=2 \mathrm{~mm}$. At the output end of the corrugations we've placed a field monitor that covers a few mesh points in $z$ and all of $r \leq a$; we use it to monitor $E_{r}(t)$ and $H_{\phi}(t)$. From these fields we obtain the outgoing power

$$
P(t)=\frac{c}{2} \int_{0}^{a} E_{r}(r, t) H_{\phi}(r, t) r d r .
$$

The energy in the pulse then is $U=\int P(t) d t$. We expect $U \approx U_{w}$, the energy lost by the bunch.

There are in total 12,500 corrugations. The bunch length in the simulation $\sigma_{z}=100 \mu \mathrm{m}$ and the mesh size is $10 \mu \mathrm{m}$. (When a coarser mesh of $20 \mu \mathrm{m}$ is used, the wake energy $U_{w}$ is not much affected, though the pulse energy $U$ begins to deviate.) In Fig. 4 we display the pattern of transient electric fields, generated by the beam, as it begins its traversal through a corrugated beam pipe. The beam on axis (at bottom right in the plot) is moving to the right 
at speed $c$. The corrugated wall is at the top. In a standard run, the field is calculated over a window that reaches $8 \mathrm{~cm}$ behind the bunch, in order that a pulse as long as $8 \mathrm{~cm}$ can be captured. A whole run takes several hours on a Windows desktop machine.

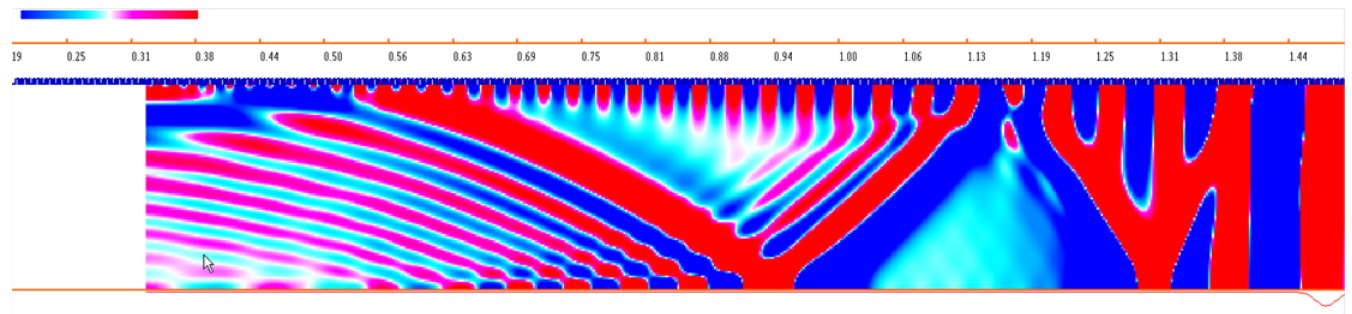

FIG. 4. Pattern of transient electric fields, generated by a beam, as it begins its traversal through a corrugated beam pipe (ECHO output). The beam on axis (at bottom right in the plot) is moving to the right at speed $c$.

The $E_{r}$ field recorded at the monitor at $r=1.5 \mathrm{~mm}$, is shown in Fig. 5 (the left plot). The monitor is at $z=50.2 \mathrm{~mm}$. Until the bunch reaches it, at $c t=50.2 \mathrm{~mm}$, there is no signal; for $\Delta c t=2.7 \mathrm{~mm}, E_{r}$ oscillates with amplitude $32 \mathrm{MV} / \mathrm{m}$; finally, the field drops to near zero again. The absolute value of the Fourier transform of the field $\left|\tilde{E}_{r}\right|$ is plotted on the right. We see that the spectrum is quite narrow, with a peak at $f=290 \mathrm{GHz}$.

Next we consider the tapered structure, with parameters given in Table I. For this structure the beam pipe radius decreases from $a=2 \mathrm{~mm}$ at the beginning to $a=1 \mathrm{~mm}$ at the end. In Fig. 6, in the left plot, we give $E_{r}$ at the monitor. We see that the pulse has an amplitude that is again nearly constant, $\sim 70 \mathrm{MV} / \mathrm{m}$, and its length $\ell \sim 3.7 \mathrm{~mm}$. The energy in the pulse $U=2.30 \mathrm{~mJ}$. The analytical approximation (Eq. 5) gives $U_{w}=2.15 \mathrm{~mJ}$. The agreement is quite good, with the discrepancy giving a measure of the accuracy of the numerical result. 

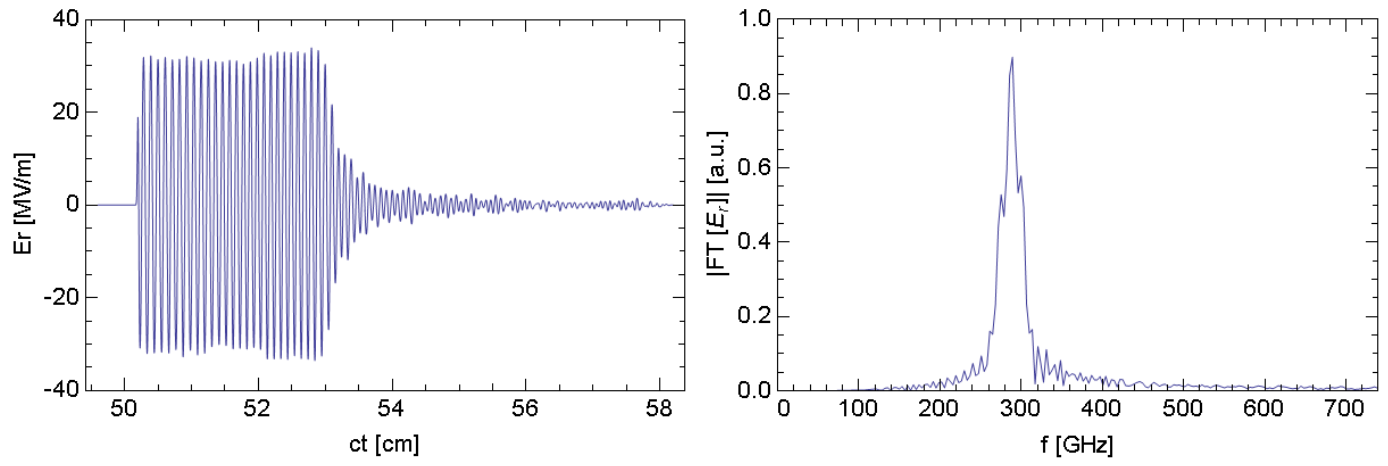

FIG. 5. For untapered structure, with $a=2 \mathrm{~mm}: E_{r}$ at monitor, at $r=1.5 \mathrm{~mm}$ (left plot); note that pulse length $\ell \sim 2.7 \mathrm{~cm}$. Absolute value of Fourier transform of $E_{r}$ (right plot).
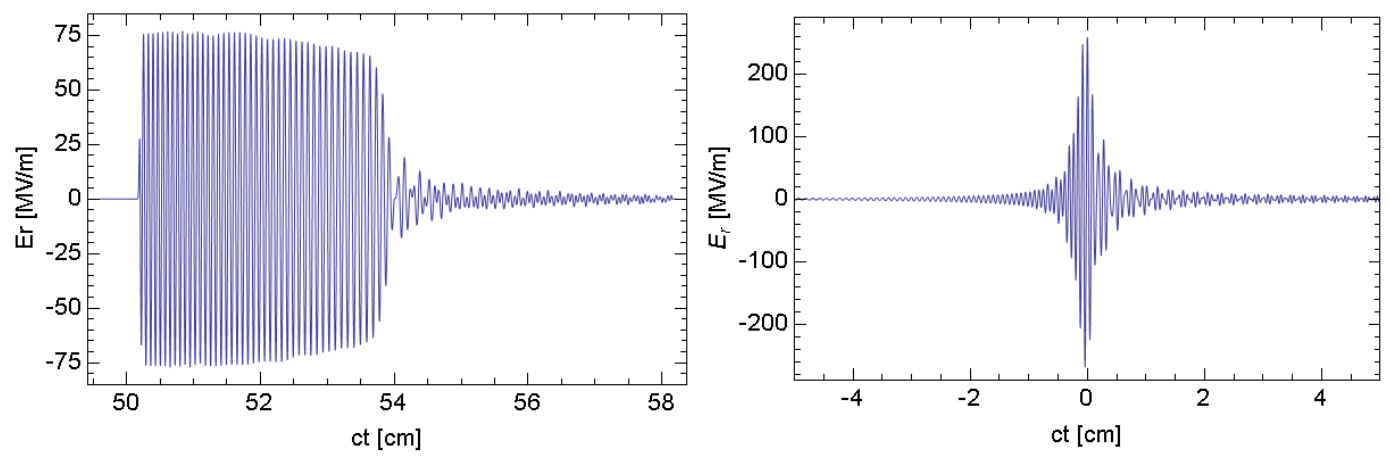

FIG. 6. For tapered structure $E_{r}$ at monitor, at $r=0.75 \mathrm{~mm}$ : before compression (left plot), and after (right plot, discussed below).

In Fig. 7 we plot the amplitude of the Fourier transform of $E_{r}$ (left figure) and its phase (in radians, on the right). We see that the spectrum width and central frequency are $(\Delta f)_{f w}=110 \mathrm{GHz}$ and $f_{c e n}=360 \mathrm{GHz}$; which are close to the analytical estimates from above, $(\Delta f)_{f w}=125 \mathrm{GHz}, f_{c e n}=350 \mathrm{GHz}$. In the phase, over the spectrum core, we see a clear quadratic dependence, 
showing that there is a rather linear frequency chirp in the pulse. The dashes in the curve give the quadratic fit over $[260,480] \mathrm{GHz}$. The effective chirp parameter is $\beta / c^{2}=0.039 \mathrm{~mm}^{-2}$. To estimate the effectiveness of a compressor that can remove a linear chirp, we subtract the fitted quadratic dependence from the phase, and perform the inverse Fourier transform. The result is shown in Fig. 6, the right plot. The final pulse length (FWHM) is $\ell_{f}=3.1 \mathrm{~mm}$, and the ratio of initial to final length is $f_{c}=12$.
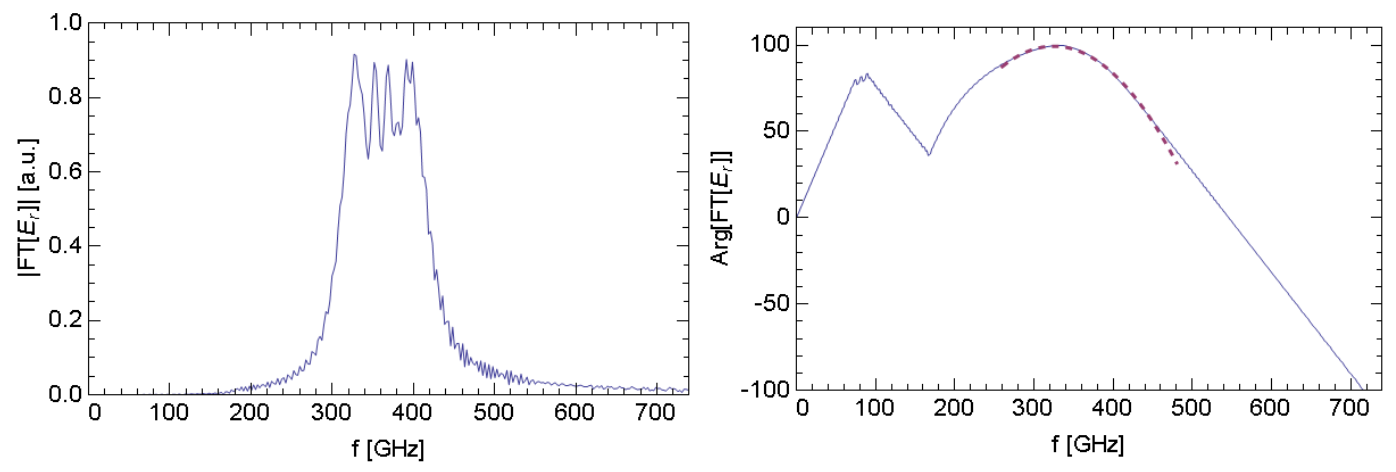

FIG. 7. For tapered structure: Absolute value of the Fourier transform $\left|\tilde{E}_{r}\right|$ (left plot) and the phase (in radians, right plot). Red dashes give quadratic fit over [260, 480] GHz.

\section{RESISTIVE WALL LOSSES}

Until now we have assumed that the walls of our corrugated pipe are perfectly conducting. With wall losses some of the wake energy lost by the beam will be lost into the walls. We present here an analytical estimate of the wall losses. Recently the program ECHO has been upgraded to included also the effect of wall losses [16]. For our tapered, corrugated pipe we will perform simulations including wall losses and then compare with the analytical results. 
TABLE II. Radiation Properties (Numerical Results).

\begin{tabular}{||l|c||}
\hline \hline Frequency $f, \mathrm{THz}$ & $0.30-0.44$ \\
Pulse energy $U, \mathrm{~mJ}$ & 2.3 \\
Pre-compression: & \\
Peak power $P, \mathrm{MW}$ & 40 \\
Pulse length $\ell, \mathrm{cm}$ & 3.5 \\
Post-compression: & \\
Peak power $P, \mathrm{MW}$ & 490 \\
Pulse length $\ell, \mathrm{cm}$ & 0.3 \\
\hline
\end{tabular}

It can be shown that the mode fields vary linearly with radius: $E_{r}=H_{\phi}=$ $H_{0} r / a$. Then the energy flow, at a given position $z$, is given by an integral over the beam pipe cross-section

$$
\Sigma=\frac{c}{8 \pi} \int\left|H_{\phi}\right|^{2} 2 \pi r d r=\frac{c}{16} a^{2} H_{0}^{2}
$$

The energy dissipation per unit length involves a line integral (azimuthally) along the wall

$$
\mathcal{P}=\frac{c}{8 \pi} \zeta^{\prime} \int\left|H_{0}\right|^{2} d l=\frac{c}{4} \zeta^{\prime} a H_{0}^{2},
$$

with $\zeta^{\prime}=(k c / 8 \pi \sigma)^{1 / 2}$, and $\sigma$ the conductivity of the metal walls. Note, however, that the length of wall boundary per unit length of corrugated pipe is $\alpha=1+(2 \delta / p)$ (for the structure of Table I, $\alpha=3.5$ ). Combining the three factors, we obtain the dissipation length, i.e. the distance over which the energy drops by $1 / e$ :

$$
L_{D}=\frac{\Sigma}{\alpha \mathcal{P}}=\frac{a}{4 \zeta^{\prime}(1+2 \delta / p)} .
$$


If we take a resistive, corrugated pipe of length $L$, with parameters that vary gradually along its length, and excite it by a bunch as described here, then the fraction of energy (lost by the beam) that ends up in the radiation pulse when it reaches the end is approximately

$$
\eta=\frac{1}{L} \int_{0}^{L} e^{-z / L_{D}(z)} d z .
$$

In the special case where the pipe dimensions are unchanging with $z, \eta=$ $\frac{L_{D}}{L}\left(1-e^{-L / L_{D}}\right)$.

Let us consider a beam pipe made of $\mathrm{Cu}$, with $\sigma=5.9 \times 10^{7} \Omega^{-1} \mathrm{~m}^{-1}$. Taking our nominal structure (Table I), but untapered with $a=2 \mathrm{~mm}$, and performing an ECHO calculation (with losses) we find that the fraction of energy that reaches the end, $\eta=0.55$; the analytical result is $\eta=0.56$, in good agreement. We then performed an ECHO calculation for our nominal, tapered structure. The radiation pulse with losses, both before and after ideal compression is shown in Fig. 8. We clearly see that, before compression, the amplitude of the pulse decreases dramatically with time. After compression the peak amplitude is $E_{r}=150 \mathrm{MV} / \mathrm{m} v s .235 \mathrm{MV} / \mathrm{m}$ in the case of no losses (Fig. 6, on the right); the energy in the pulse is $U=1.10 \mathrm{~mJ}$ vs. $2.30 \mathrm{~mJ}$ with no losses. Here $\eta=0.44$, and the analytical estimate of energy in the pulse, $Q^{2} L \eta\langle\kappa\rangle=1.10 \mathrm{~mJ}$, is in excellent agreement with the numerical result.

If we increase the period of corrugation (keeping $g=p / 2$ ) and keep other parameters fixed, then the wall length along the surface per unit length along the axis - $\alpha$-is reduced, which increases the fraction of energy that makes it to the end $-\eta$. However, the loss factor $\kappa$, which gives the strength of excitation, is reduced (and no longer given by Eq. 4). There is an optimum that maximizes the energy in the pulse at the end of the structure. If we increase $p$ to $160 \mu \mathrm{m}$, then $\alpha=1.6$, and $\eta=0.64$. ECHO finds that the loss factor is reduced by $\sim 20 \%$, and the energy in the pulse at the end, $U=1.20 \mathrm{~mJ}$-slightly larger 

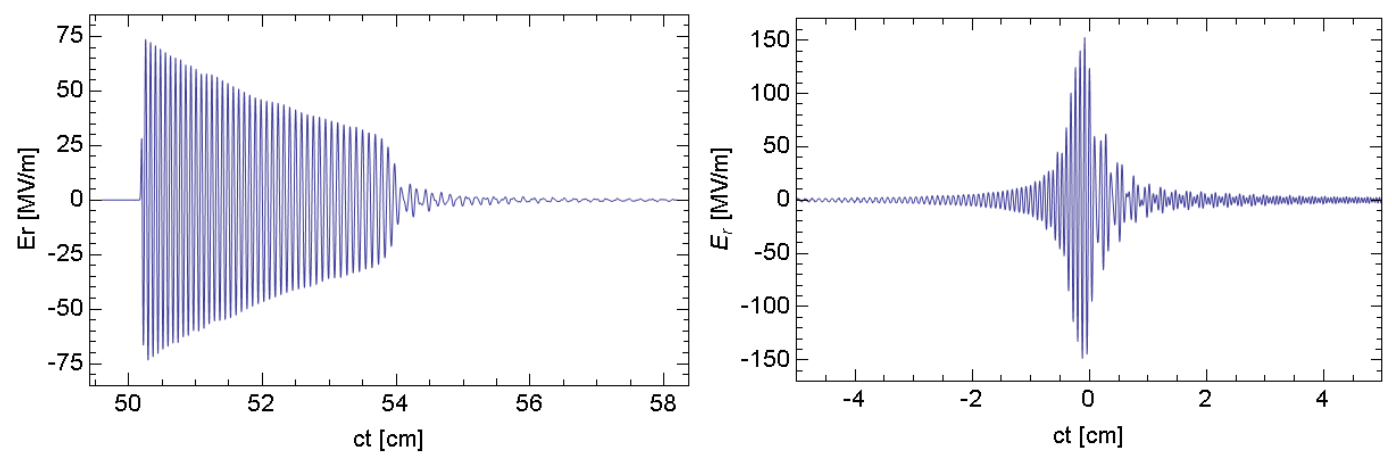

FIG. 8. For tapered structure, including resistive wall losses $(\mathrm{Cu}): E_{r}$ at monitor, at $r=0.75 \mathrm{~mm}$ before (left plot) and after (right plot) compression.

than the $1.10 \mathrm{~mJ}$ obtained for the nominal structure.

At liquid nitrogen temperature, $77 \mathrm{~K}, \sigma$ for $\mathrm{Cu}$ is increased a by factor of 7.2 [17]. Placing the structure in a liquid nitrogen bath will thus reduce the wall losses. At $77 \mathrm{~K}$, the estimate for the $p=160 \mu \mathrm{m}$ example: $\eta=0.83$ and $U=1.55 \mathrm{~mJ}$.

\section{REACHING TO 1 THZ}

How can we reach $1 \mathrm{THz}$ with the corrugated pipe, and how can we maximize the energy in the pulse? For scaling, we see that the frequency $f \sim(a \delta)^{-1 / 2}$, wake energy $U_{w} \sim L / a^{2}$, damping length $\left(L_{D} / L\right) \sim a^{5 / 4} \delta^{1 / 4} / L$, pulse length before compression $\ell \sim \delta L / a$, and after compression $\ell_{f} \sim(a \delta)^{1 / 2}$. $U_{w}$ does not depend on corrugation size. If we reduce $\delta$ (and also $p, g$ ) by factor $A$, the frequency increases $f \rightarrow \sqrt{A} f$.

To reach $1 \mathrm{THz}$, we consider the beam pipe radius $a$ varying linearly from $1-0.5 \mathrm{~mm}$ along the structure, take pipe length $L=12.5 \mathrm{~cm}$, corrugation depth $\delta=20 \mu \mathrm{m}$, corrugation period $p=60 \mu \mathrm{m}$, and bunch length 
$\sigma_{z}=40 \mu \mathrm{m}$. We've run Echo for this example assuming $\mathrm{Cu}$ walls (at room temperature). We obtain a pulse at the output end of the structure that has peak field at the wall of $E_{r}=260 \mathrm{MV} / \mathrm{m}$ and a pulse length $\ell=5.9 \mathrm{~mm}$. The wake energy $U_{w}=2.2 \mathrm{~mJ}$ and the pulse energy $U=1.6 \mathrm{~mJ}$. In Fig. 9 we plot (at $r=0.375 \mathrm{~mm})\left|\tilde{E}_{r}(\omega)\right|$ (left frame) and $E_{r}(t)$ after ideal compression (right frame). The spectrum has a central frequency of $0.9 \mathrm{THz}$, with FWHM of $0.25 \mathrm{THz}$. Post compression the peak power $P=1.0 \mathrm{GW}$ and the pulse length $\ell=1.1 \mathrm{~mm}$. The compression factor is 5.4 .
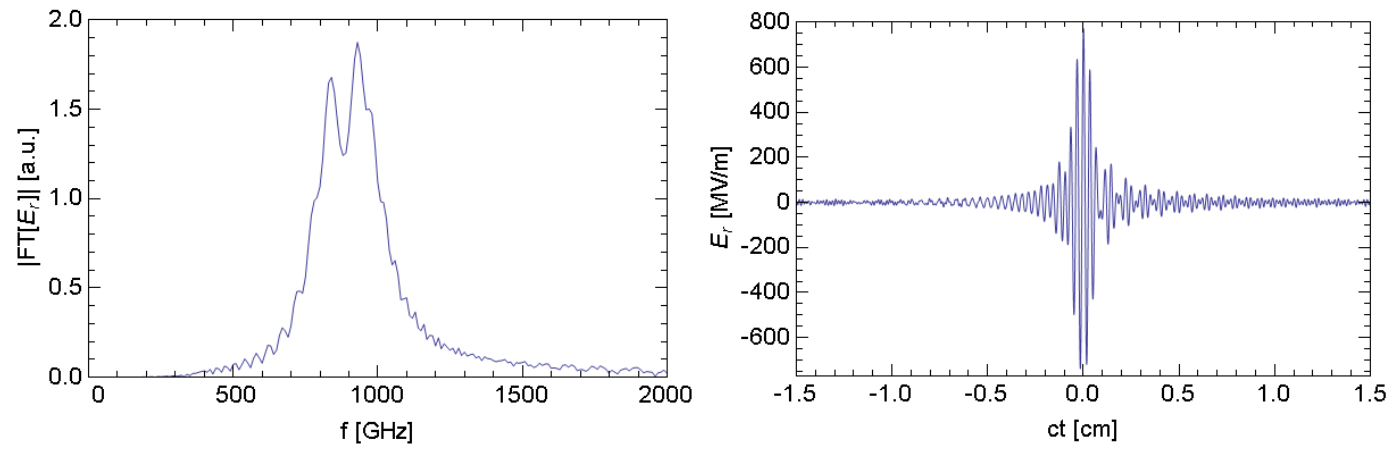

FIG. 9. For $1 \mathrm{THz}$ structure, at $r=0.375 \mathrm{~mm}$ : Fourier transform of $E_{r}$ at monitor (left plot) and $E_{r}$ after ideal compression (right plot).

One issue that might be of concern is that the fields on the walls are quite large, $260 \mathrm{MV} / \mathrm{m}$, and the corrugations have sharp edges. We believe, however, that the radiated pulse properties will be relatively unchanged if the rectangular corrugation is replaced by a sinusoidal one that represents the first term in the Fourier series of the original structure. This modification, combined with the fact that the radiation pulse is of very short length, leads us to anticipate no damage to the walls, though this question needs more study.

What is the practical limit to the frequency that can be generated by such a structure? Consider a structure with fixed $a=1 \mathrm{~mm}$ and $L=12.5 \mathrm{~cm}$ (as 
in the previous example), but with the corrugation dimensions reduced by a further factor of 10 , so that e.g. $\delta=2 \mu \mathrm{m}$. If we also reduce the bunch length by $\sqrt{10}$ to $\sigma_{z}=12.5 \mu \mathrm{m}$, we should in theory be able to reach $f=2.8 \mathrm{THz}$. The wall losses and energy in the pulse should be relatively unchanged from the previous example, and the pulse length, even without compression, should be relatively short, $\ell \sim 0.5 \mathrm{~mm}$. A practical question is, can such small corrugations be produced reasonably accurately? Also, note that the catch-up distance $a^{2} / 2 \sigma_{z} \sim 4 \mathrm{~cm}$, which is a third of the pipe length, meaning that the transient fields start to become important and the analytical formulas begin to lose their validity.

\section{CONCLUSION AND DISCUSSION}

We have analyzed and studied through numerical simulation the use of an electron bunch to drive a metallic beam pipe with small corrugations for the purpose of generating terahertz radiation. For the case of a pipe with dimensions that do not change along its length, we have shown that-with reasonable parameters - one can generate a narrow-band radiation pulse with frequency $\sim 1 \mathrm{THz}$, and high total energy: 1 or 2 milli-Joules. The pulse length, however, tends to be rather long, on the order of 10's of ps. We have further shown that: (i) by using a tapered pipe, one generates a pulse with a frequency chirp, and (ii) if the chirped pulse then passes through a dispersive device (a "compressor"), one can end up with a final pulse only a few ps in length, at the cost of a wider bandwidth.

We have shown that wall losses in the corrugated pipe tend to be significant and need to be considered in the calculations. The energy in the radiation pulse is given by the (wake) energy lost by the bunch minus the energy lost in the walls. For a given corrugation amplitude there is an optimal corrugation 
period: if it is shorter, the wall losses increase; if it is longer the wake energy decreases. We have found that analytical estimates of resistive wall losses and pulse energy agree very well with numerical results.

Among the practical problems that need to be addressed before this method of generating $\mathrm{THz}$ radiation can be realized are: (1) (in the chirped pulse case) how to design a real $\mathrm{THz}$ pulse compressor, (2) how to extract the $\mathrm{THz}$ pulse from the pipe without destroying its properties, and (3) how to guide and focus

the pulse to an experiment. Recently Geloni, et al, have found that a round metallic pipe of radius of several cm's, with a periodic array of shallow irises, can be used to transport $\mathrm{THz}$ radiation with little loss [15]. We can imagine that a smooth metallic pipe that is gradually tapered from the end of our corrugated structure to such a transport pipe may be an effective transition piece for transporting the $\mathrm{THz}$ pulse, though this needs more study.

One limitation of using a round, corrugated pipe to generate $\mathrm{THz}$ radiation is that once it is manufactured its frequency response is fixed. If, instead, we used two flat, corrugated plates to generate a pulse between them, the frequency could be adjusted by changing the separation of the plates. Earlier studies have found that a mode similar to that in the round pipe is generated by this configuration, though for a given aperture the excitation appears to be about a factor of two weaker (see e.g. [12]). Nevertheless, this may be an attractive alternative geometry for future studies.

\section{ACKNOWLEDGMENTS}

The authors thank V. Dolgashev for helpful discussions on the use of corrugated pipes for the generation of $\mathrm{THz}$ radiation and $\mathrm{Z}$. Huang on information 
on laser-based methods of generating $\mathrm{THz}$ radiation.

[1] D. Auston et al., Phys. Rev. Lett. 53, 1555 (1984).

[2] D. You et al., Opt. Lett. 18, 290 (1993).

[3] A. Sell et al., Opt. Lett. 33, 2767 (2008).

[4] T. Nakazato et al., Phys. Rev. Lett. 63, 1245 (2008).

[5] G. L. Carr et al., Nature 420, 153 (2002).

[6] D. Daranciang et al., Appl. Phys. Lett. 99, 141117 (2011).

[7] A. M. Cook et al., Phys. Rev. Lett. 103, 095003 (2009).

[8] I. Zagorodnov and T. Weiland, Phys. Rev. ST Accel. Beams 8, 042001 (2005).

[9] K. Bane and A. Novokhatski, The resonator impedance model of surface roughness applied to the LCLS parameters, LCLS-TN- 99-1 (SLAC, 1999).

[10] K. Bane and G. Stupakov, in Proc. of LINAC00, Monterey, CA (2000) pp. $92-94$.

[11] A. Chao, "Physics of collective beam instabilities in high energy accelerators," (John Wiley \& Sons, 1993) p. 121.

[12] K. Bane and G. Stupakov, Phys. Rev. ST Accel. Beams 6, 024401 (2003).

[13] A. Chao, "Physics of collective beam instabilities in high energy accelerators," (John Wiley \& Sons, 1993) p. 41.

[14] Lectures by R. Trebino at https://public.me.com/ricktrebino/Ultrafast Optics/Lectures.

[15] G. Geloni, V. Kocharyan, E. Saldin, Scheme for generating and transporting THz radiation to the X-ray experimental floor at the LCLS baseline, DESY 11-134 (DESY, 2011).

[16] A. Tsakanian, M. Dohlus, I. Zagorodnov, in Proc. of EPAC08, Genoa, Italy (2008) pp. 1709-1711. 
[17] W. M. Haynes, ed., "CRC handbook of chemistry and physics, 92nd edition," (CRC Press, 2011) pp. 12-41. 